\title{
Correction to: From materials to systems: a multiscale analysis of nanomagnetic switching
}

\author{
Yunkun Xie ${ }^{1}$ (1) Jianhua $\mathrm{Ma}^{1} \cdot$ Samiran Ganguly ${ }^{1} \cdot$ Avik W. Ghosh $^{1,2}$
}

Published online: 23 February 2018

(c) Springer Science+Business Media, LLC, part of Springer Nature 2018

\section{Correction to: J Comput Electron (2017) 16:1201-1226 https://doi.org/10.1007/s10825-017-1054-z}

The original version of this article unfortunately contained an error. The authors would like to correct the error with this erratum.

Unfortunately, in the original publication of the article, we left out a NSF award in the acknowledgement that funded part of the work presented in this article. We presented the corrected acknowledgement here.
Acknowledgements We acknowledge discussions with Prof. W. H. Butler on Heusler studies, I. Rungger and S. Sanvito from the Smeagol team for ab-initio transport calculations, P. Visscher and B. BehinAein on Fokker-Planck and write error study in STT-MRAM. We also acknowledge the support from Oak Ridge National Laboratory (ORNL) center for nanophase materials sciences (CNMS) on computational resources. This work was funded over the years by NSF-DMREF No. 1235230, DARPA, NSF-SHF No. CCF1514219, SRC-NRI and IBM Fellowship.

The original article can be found online at https://doi.org/10.1007/ s10825-017-1054-z.

Yunkun Xie

yx3ga@ virginia.edu

1 Department of Electrical and Computer Engineering, University of Virginia, Charlottesville, VA 22903, USA

2 Department of Physics, University of Virginia, Charlottesville, VA 22903, USA 